\title{
Dexmedetomidine Increases the Latency of Thermal Antinociception in Rats
}

\author{
Yukako Tsutsui, DDS, PhD, and Katsuhisa Sunada, DDS, PhD \\ Department of Dental Anesthesiology, The Nippon Dental University School of Life Dentistry at Tokyo, Japan
}

Recent reports have stated that dexmedetomidine (DEX), an $\alpha_{2}$-adrenoreceptor agonist, enhances the local anesthetic effects of ropivacaine and prolongs its effective duration. However, little is known about the effect of a combination of DEX and lidocaine on anesthetic duration. Therefore, we investigated whether DEX can prolong the local anesthetic effect of lidocaine, using the thermal paw withdrawal test in Wistar rats in order to measure local anesthetic duration. We subcutaneously injected $50 \mu \mathrm{L}$ of either normal saline, $2 \%$ lidocaine, a combination of $0.5 \mu \mathrm{g} / \mathrm{kg}$ DEX and $2 \%$ lidocaine, or a combination of $2 \%$ lidocaine with 1:80,000 epinephrine into the plantar surface of the left hind paw of the rats. The plantar region was stimulated using heat. We measured the perceived acute pain according to paw movement in response to stimulation. We found DEX significantly prolonged the paw withdrawal latency of lidocaine. Moreover, we found that DEX can prolong the local anesthetic duration of lidocaine as much as 1:80,000 epinephrine, up to 35 minutes after injection. In conclusion, this study concluded that a combination of DEX and lidocaine may be useful as a local anesthetic, similar to a combination of epinephrine and lidocaine, for short procedures.

Key Words: Dexmedetomidine; Lidocaine.

D entists routinely use added low-dose epinephrine to prolong the duration and efficacy of dental local anesthesia. However, epinephrine may produce unwanted hypertension and tachycardia, particularly in those with cardiovascular compromise. Therefore, alternative vasoconstrictors added to dental local anesthetics would be desirable.

Dexmedetomidine (DEX) is a selective $\alpha_{2}$-adrenoreceptor agonist used for intravenous procedural sedation and sedation of intubated patients in intensive care units. It has recently been reported that DEX enhances local anesthetic effects and prolongs the duration of their efficacy on the peripheral nerves. ${ }^{1-4}$ These effects were shown to be dose dependent and peripherally mediated. Kosugi et $\mathrm{al}^{5}$ have reported that a high dose of DEX inhibits the compound action potential in frog sciatic nerves more strongly than does clonidine. The effect was not reversed by an $\alpha_{2}$-adrenoreceptor antagonist. Additionally, it has been reported that

Received November 10, 2016; accepted for publication April 24, 2017.

Address correspondence to Dr Yukako Tsutsui, DDS, PhD, Department of Dental Anesthesiology, The Nippon Dental University School of Life Dentistry at Tokyo, 1-9-20 Fujimi, Chiyodaku, Tokyo 102-8159, Japan; tsutsuiy@tky.ndu.ac.jp.

Anesth Prog 65:230-234 2018 | DOI 10.2344/anpr-65-01-08

(C) 2018 by the American Dental Society of Anesthesiology
DEX blocks tetrodotoxin-resistant $\mathrm{Na}^{+}$channels directly. ${ }^{6}$ Tetrodotoxin-resistant $\mathrm{Na}^{+}$channels are key receptor elements in the transmission of nociceptive sensory information, as nociceptive signals depend on the propagation of action potentials through tetrodotoxinresistant $\mathrm{Na}^{+}$channels. ${ }^{7}$ Indeed, local anesthetics prevent pain by directly blocking $\mathrm{Na}^{+}$channels. ${ }^{8}$ Many dentists prefer to use lidocaine, which acts as a $\mathrm{Na}^{+}$ channel blocker, as a dental local anesthetic in the clinic.

Consequently, we hypothesized that a combination of DEX and lidocaine would prolong the dental local anesthetic effect achieved by lidocaine alone. Thus, in this study, we tested whether a combination of DEX and lidocaine could enhance the duration of sensory blockade as compared with lidocaine alone. In addition, we tested whether DEX could prolong the effect of lidocaine as much as epinephrine using the thermal paw withdrawal test in rats.

\section{METHODS}

All experimental procedures and protocols used in the present study were approved by the Animal Care and Use Committee at The Nippon Dental University. All procedures conformed to the Guide for the Care and Use of Laboratory Animals. The experiments were performed using adult male Wistar rats $(N=40)$. The rats were 
acclimated in an animal room with water and food ad libitum for 1 week before the experiment. All rats weighed approximately $250 \mathrm{~g}(250 \pm 18 \mathrm{~g})$.

DEX hydrochloride was purchased from SigmaAldrich (St Louis, Mo). A solution of $2 \%$ lidocaine (L) and a combination of $2 \%$ lidocaine with 1:80,000 epinephrine (EL) was purchased from AstraZeneca (Osaka, Japan). DEX was dissolved in the L solution at a concentration of $2.5 \mu \mathrm{g} / \mathrm{mL}$ to prepare the lidocaine combined with $0.5 \mu \mathrm{g} / \mathrm{kg}$ DEX (DL) solution. Brummett et $\mathrm{al}^{4}$ have previously reported that DEX added to ropivacaine increased the duration of analgesia to a heat stimulus in a dose-dependent fashion. In their report, the lowest effective dose of DEX was $0.5 \mu \mathrm{g} / \mathrm{kg}$. Therefore, we selected a combination of $0.5 \mu \mathrm{g} / \mathrm{kg}$ DEX and 2\% lidocaine for use in this study. The $\mathrm{pH}$ of the L solution $(5.88 \pm 0.02)$ was used as the standard to which all the solutions were standardized. The EL solution contained $12.5 \mu \mathrm{g}$ epinephrine and $20 \mathrm{mg}$ lidocaine in $1 \mathrm{~mL}$ solution.

Paw withdrawal latency was used to measure the local anesthetic effect, ${ }^{9,10}$ using a modified device based on a design by Hargreaves et al. ${ }^{9}$ We subcutaneously injected $50 \mu \mathrm{l}$ of either normal saline (NS), L, DL, or EL solutions into the plantar surface of the rat's left hind paw using a 31-gauge needle. The rat's left hind paw was injected with one solution, once only per experiment, with 10 rats per group. Then, the rat was placed in a wire net container. The container was placed on a heated glass base. We stimulated the left hind paw plantar region with intense heat by placing the plantar region of the left hind paw on the infrared radiant stimulator (Ugo Basile, Collegeville, Pa), which resulted in a skin temperature of $65^{\circ} \mathrm{C}$ after 10 seconds. We measured perceived acute pain by paw movement in response to stimulation. When the rat moved its left hind paw because of pain, the infrared radiant heater turned off automatically. Paw withdrawal latency was measured from the start of contact with the infrared radiant heat until paw withdrawal. The paw withdrawal latency was tested only once at each time point, at 5minute intervals. We set the cutoff time for withdrawal at 10 seconds.

All values of withdrawal latency are expressed as mean $\pm \mathrm{SD}$. A repeated-measures analysis of variance evaluated the effects on paw withdrawal latency, and Bonferroni post hoc test were used to compare paw withdrawal latency for 4 treatments (NA, L, DL, and EL) and the time dependency (baseline to 40 minutes after injection) of the effects. Values of $p<0.05$ were considered statistically significant. All statistical analyses were performed with Microsoft Excel 2013 (Microsoft, Redmond, Wash).

\section{RESULTS}

As shown in the Figure and the Table, we monitored the paw withdrawal latency of rats that had been subcutaneously injected with NS, L, DL, or EL solution into their left hind paw plantar region. The paw withdrawal latencies at each time point after injection of L, DL, and EL solutions were significantly longer than that after injection of NS solution. However, the paw withdrawal latency at 40 minutes after $\mathrm{L}$ solution injection was not significantly longer than that after injection of NS solution. In addition, the paw withdrawal latencies from 25 to 40 minutes after DL or EL solution were significantly longer than that after injection of $\mathrm{L}$ solution. Moreover, the paw withdrawal latencies at 35 and 40 minutes after EL solution were significantly longer than that after injection of DL solution.

\section{DISCUSSION}

In this study, we measured the paw withdrawal latency of rats treated with $\mathrm{L}$, DL, or EL using a paw withdrawal latency test, and found that paw withdrawal latency was significantly increased following DL and EL injections as compared to $\mathrm{L}$ injection from 25 to 40 minutes after injection. Moreover, DEX prolonged the local anesthetic activity of lidocaine as effectively as epinephrine up until and including 30 minutes after injection. However, epinephrine prolonged the local anesthetic effectiveness of lidocaine longer than DEX at 35 and 40 minutes after injection. These results showed that DEX prolonged the effective local anesthetic duration of lidocaine as well as epinephrine did up to 30 minutes after injection. Our findings suggested that a combination of DEX and lidocaine can be a useful local anesthetic substitute for a combination of epinephrine and lidocaine.

Epinephrine occasionally produces adverse cardiovascular side effects, such as hypertension and tachycardia. A large increase in blood pressure and/or heart rate poses a potential problem for patients with cardiovascular disease. Additionally, DEX is known to result in bradycardia and hypotension when administered as an intravenous infusion. ${ }^{11}$ Low concentrations, such as that used in this study, may not be expected to produce these effects; or, if present, the effects may be mild. Therefore, a combination of DEX and lidocaine may be a useful local anesthetic for patients in whom the dentist chooses to not use epinephrine.

Our findings concur with a report by Memiş et al, ${ }^{12}$ who stated that the duration of analgesia was significantly increased with DEX. They reported that DEX added to lidocaine for intravenous regional anesthesia 


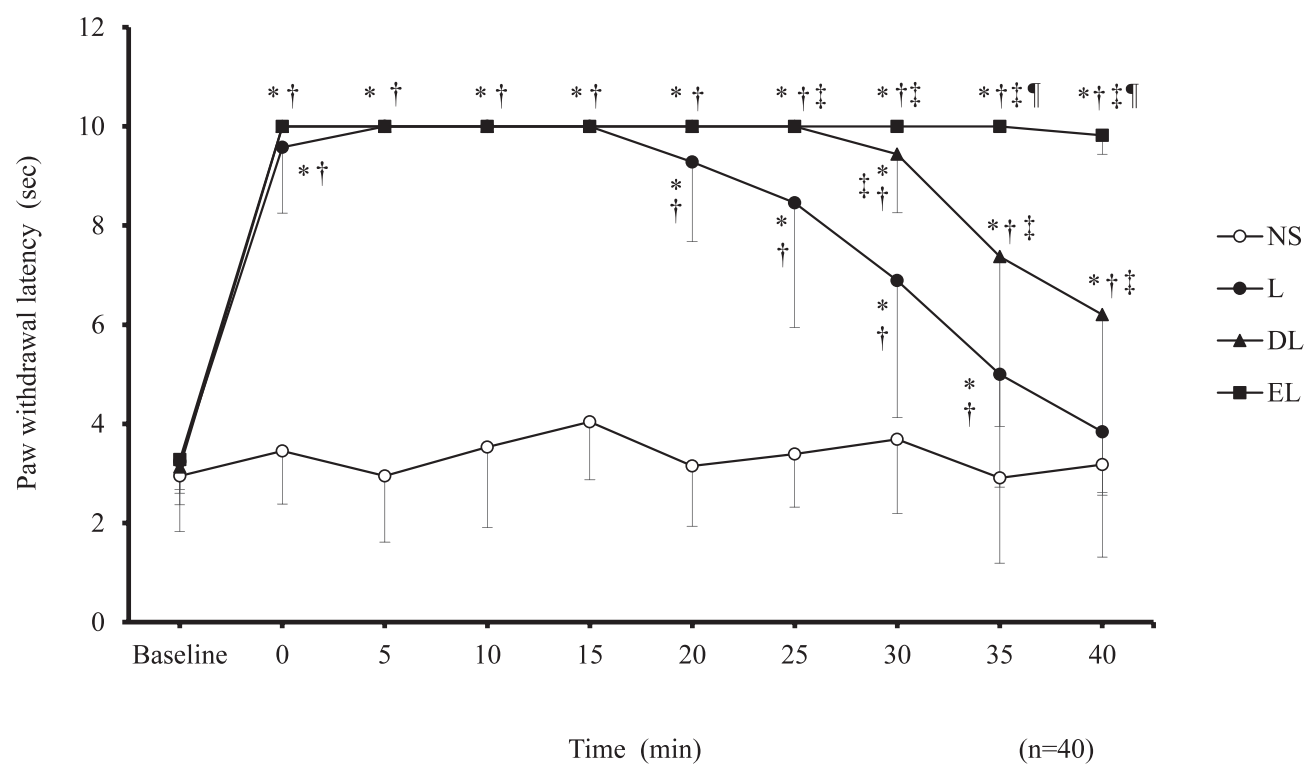

Paw withdrawal latency with NS $(n=10), 2 \%$ lidocaine $(n=10), 0.5 \mu \mathrm{g} / \mathrm{kg}$ DEX combined with $2 \%$ lidocaine $(n=10)$, and $1: 80,000$ epinephrine combined with $2 \%$ lidocaine $(n=10)$ at each time point. We monitored paw withdrawal latency from immediately after injection to 40 minutes after injection. White circles represent the NS group; black circles represent the $2 \%$ lidocaine (L) group; black triangles represent the $0.5 \mu \mathrm{g} / \mathrm{kg}$ DEX combined with $2 \%$ lidocaine (DL) group; and black squares represent the 1:80,000 epinephrine combined with $2 \%$ lidocaine (EL) group. $*$ indicates significant difference from baseline at each time point; $\dagger$, significant difference from normal saline at each time points; $\$$, significant difference from $2 \%$ lidocaine at each time point; 9 , significant difference from $0.5 \mu \mathrm{g} / \mathrm{kg} \mathrm{DEX}+2 \%$ lidocaine at each time point.

delayed the onset of tourniquet pain and reduced the intraoperative and postoperative analgesic requirement. Moreover, Yamane et $\mathrm{l}^{13}$ reported that a combination of DEX and lidocaine increased the current perception threshold in human oral mucosa. They submucosally injected a combination of DEX and lidocaine solution in humans. In their report, they concluded that a combination of DEX and lidocaine enhanced the local anesthetic effectiveness of lidocaine. Based on the findings of our study and these reports, we suggest that a combination of DEX and lidocaine will be more useful as a dental local anesthetic than lidocaine alone in humans. However, both our own and these other reports were limited in terms of investigating the mechanisms underlying the prolongation. Other studies have reported several possible mechanisms. One is an analgesic effect exerted via the $\alpha_{2}$-adrenoreceptor. The $\alpha_{2}$-adrenoceptors are involved in the regulation of pain transmission, and agonists of the $\alpha_{2}$-adrenoreceptor mediate antinociception. ${ }^{6,14-17}$ Another possibility is the effect of vasoconstriction around the injection site. A peripheral mechanism of DEX has been demonstrated to involve vasoconstriction via the $\alpha_{2 \mathrm{~B}}$-adrenergic receptor. ${ }^{18,19}$ Indeed, both possible mechanisms support the assertion that DEX enhances the local anesthetic effect by prolonging the effective duration. In the future, we would like to investigate the mechanisms underlying the prolongation effects.

Additionally, we compared DEX with epinephrine in this study. Epinephrine is commonly used as a vasoconstrictor with lidocaine in dental local anesthetics. In our study, DEX prolonged the effect of lidocaine, similarly to epinephrine, up to 30 minutes after injection. This suggested that a combination of DEX and lidocaine could be used as a dental local anesthetic, analogous to a combination of epinephrine and lidocaine for short dental treatments. Repeat injection, or use of a different concentration of DEX, might further improve the efficacy and duration profile and is an area for further investigation.

In the present study, all rats experienced prolongation of the effect of local anesthesia when given a combination of DEX and lidocaine. The data implied that the effects of DEX were predominantly exerted at the peripheral nerve level as the administered concentration was low. However, we did not investigate a dosedependent effect of DEX. Normally, a higher dose of DEX would provide a general sedative effect. In future, investigations should consider whether a combination of DEX and lidocaine has a general sedative effect by performing a dose-series study.

In conclusion, we validated that DEX prolongs the local anesthetic duration of lidocaine in the paw 
Paw Withdrawal Latency Values at Each Time Point From Immediately to 40 Minutes After Injection*

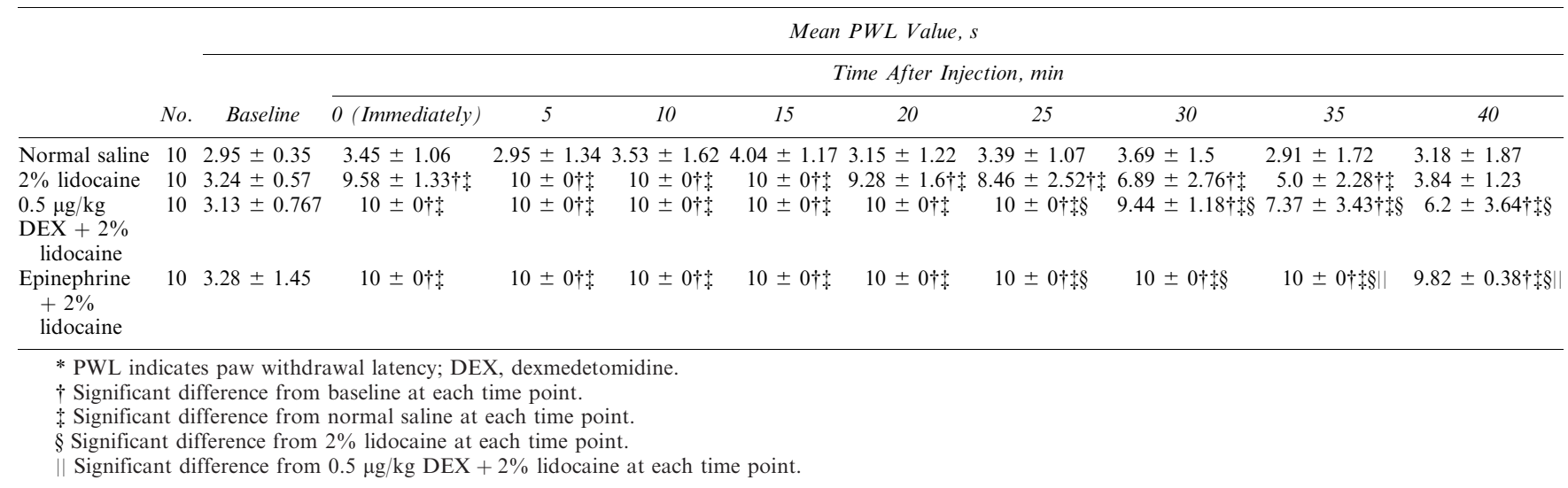

withdrawal latency test. Moreover, we found that DEX added to lidocaine prolongs peripheral nerve block as much as epinephrine, up to 30 minutes after injection. These data suggest that a combination of DEX and lidocaine may be a useful local anesthetic, similar to a combination of epinephrine and lidocaine, for short dental procedures.

\section{ACKNOWLEDGMENT}

This study was supported by Grant-in-Aid for Scientific Research, Young Scientists of Japan (B) 26861755.

\section{REFERENCES}

1. Brummett CM, Norat MA, Palmisano JM, Lydic R. Perineural administration of dexmedetomidine in combination with bupivacaine enhances sensory and motor blockade in sciatic nerve block without inducing neurotoxicity in rat. Anesthesiology. 2008;109:502-511.

2. Yoshitomi T, Kohjitani A, Maeda S, Higuchi $H$, Shimada M, Miyawaki T. Dexmedetomidine enhances the local anesthetic action of lidocaine via an alpha-2A adrenoceptor. Anesth Analg. 2008;107:96-101.

3. Brummett CM, Williams BA. Additives to local anesthetics for peripheral nerve blockade. Int Anesthesiol Clin. 2011;49:104-116.

4. Brummett CM, Padda AK, Amodeo FS, Welch KB, Lydic R. Perineural dexmedetomidine added to ropivacaine causes a dose-dependent increase in the duration of thermal antinociception in sciatic nerve block in rat. Anesthesiology. 2009;111:1111-1119.

5. Kosugi T, Mizuta K, Fujita T, Nakashima M, Kumamoto E. High concentrations of dexmedetomidine inhibit compound action potentials in frog sciatic nerves without $\alpha 2$ adrenoceptor activation. Brit J Pharmacol. 2010; 160:1662-1676.
6. Oda A, Iida H, Tanahashi S, Osawa Y, Yamaguchi S, Dohi S. Effects of alpha2-adrenoceptor agonists on tetrodotoxin-resistant $\mathrm{Na}^{+}$channels in rat dorsal root ganglion neurons. Eur J Anaesthesiol. 2007;24:934-941.

7. Ogata N, Ohishi Y. Molecular diversity of structure and function of the voltage-gated $\mathrm{Na}^{+}$channels. Jpn J Pharmacol. 2002;88:365-377.

8. Fozzard HA, Lee PJ, Lipkind GM. Mechanism of local anesthetic drug action on voltage-gated sodium channels. Curr Pharm Des. 2005;11:2671-2686.

9. Hargreaves K, Dubner R, Brown F, Flores C, Joris J. A new and sensitive method for measuring thermal nociception in cutaneous hyperalgesia. Pain. 1988;32:77-88.

10. Yamamoto S, Ohsawa M, Ono H. Contribution of TRPV1 receptor-expressing fibers to spinal ventral root afterdischarges and mechanical hyperalgesia in a spared nerve injury (SNI) rat model. J Pharmacol Sci. 2013;121:9-16.

11. Ebert TJ, Hall JE, Barney JA, Uhrich TD, Colinco MD. The effects of increasing plasma concentrations of dexmedetomidine in humans. Anesthesiology. 2000:93:382-394.

12. Memiş D, Turan A, Karamanlioğlu B, Pamukçu Z, Kurt I. Adding dexmedetomidine to lidocaine for intravenous regional anesthesia. Anesth Analg. 2004;98:835-840.

13. Yamane A, Higuchi H, Tomoyasu Y, Ishii-Maruhama M, Maeda S, Miyawaki T. Effect of dexmedetomidine injected into the oral mucosa in combination with lidocaine on local anesthetic potency in humans: a crossover double-blind study. J Oral Maxillofac Surg. 2015;73:616-621.

14. Hunter JC, Fontana DJ, Hedley LR, et al. Assessment of the role of alpha2-adrenoceptor subtypes in the antinociceptive, sedative and hypothermic action of dexmedetomidine in transgenic mice. Br J Pharmacol. 1997;122:1339-1344.

15. Stone LS, MacMillan LB, Kitto KF, Limbird LE, Wilcox GL. The alpha2a adrenergic receptor subtype mediates spinal analgesia evoked by alpha 2 agonists and is necessary for spinal adrenergic-opioid synergy. J Neurosci. 1997;17:7157-7165.

16. Fairbanks CA, Wilcox GL. Moxonidine, a selective alpha2-adrenergic and imidazoline receptor agonist, produces spinal antinociception in mice. J Pharmacol Exp Ther. 1999; 290:403-412. 
17. Gaumann D, Forster A, Griessen M, Habre W, Poinsot O, Della Santa D. Comparison between clonidine and epinephrine admixture to lidocaine in brachial plexus block. Anesth Analg. 1992;75:69-74.
18. Gerlach AT, Dasta JF. Dexmedetomidine: an updated review. Ann Pharmacother. 2007;41:245-252.

19. Coursin DB, Coursin DB, Maccioli GA. Dexmedetomidine. Curr Opin Crit Care. 2001;7:221-226. 